\title{
Modelling Intermittently Present Features Using Nonlinear Point Distribution Models
}

\author{
Gerard Sanroma and Francesc Serratosa \\ Dept. of Computer Engineering and Maths, \\ Universitat Rovira i Virgili, \\ Av. Països Catalans 26, E-43007 Tarragona, Catalonia, Spain \\ gsanroma@gmail.com, francesc.serratosa@urv.cat
}

\begin{abstract}
We present in this paper a new compact model to represent a data set based on the main idea of the Point Distribution Model (PDM). Our model overcomes PDM in two aspects, first, it is not needed all the objects to have the same number of points. This is a very important feature, since in real applications, not all the landmarks are represented in the images. And second, the model captures the nonlinearity of the data set. Some research have been presented that couples both aspects separately but no model have been presented until now that couples them as a whole. A case study shows the efficiency of our model and its improvement respect the models in the literature.
\end{abstract}

Keywords: Point Distribution Models, intermittently present landmarks, missing data, statistical shape modelling, imputation.

\section{Introduction}

One of the main challenges in locating and recognising objects in images is that objects of the same class are often no identical in appearance. In such cases, deformable models, which allow for variability in the imaged objects, are appropriate [1. An important issue is limiting the shape variability to that which is consistent with the class of objects to be modelled. Point Distribution Models (PDMs) 2] is one of the most important methods used to represent such shape variability. Objects are defined by landmark points which are placed in the same way on each of the examples of the set. A statistical analysis estimates for the mean shape and the main modes of variation. These linear models have proved to be successful in capturing the variability present in some applications, however, there are some situations where they fail due to the nonlinearity of the shapes of objects that compose the class. To overcome this problem, the method called Polynomial Regression PDM (PRPDM) 3 was defined. It is based on a polynomial regression to capture the nonlinearity of the shape variability.

All the methods commented before need the same number of landmarks to be present in all the objects that compose the class. However, there are some objects that can present patterns of presence and absence in some of their features. It gives shapes with different number of points. Few research have been carried out

D. Mery and L. Rueda (Eds.): PSIVT 2007, LNCS 4872, pp. 260 273. 2007.

(C) Springer-Verlag Berlin Heidelberg 2007 
that considers intermittently present landmarks. The Structured PDM (SPDM) 4. by M. Rogers and J. Graham is a method based on the well known PDM augmented with a vector informing the inclusion or exclusion of the landmark. This method was proven to capture the variability and the presence of the landmarks on shapes both in artificial images and in electron microscope images of diabetic nerve capillaries.

In this paper, we present a new model that permits intermittently present landmarks as in SPDM and allows to capture nonlinear variability as in the PRPDM. We have called it Polynomial Regression Structured Point Distribution Model (PR-SPDM) since we consider that both problems have been solved; nonlinear variability and intermittently present landmarks.

In section 2 we present the topic of modelling intermittently present landmarks and describe an existing approach. In section 3 we describe an approach to model nonlinear variability in shapes. In section 4 we present our approach that allows for shapes with intermittently present landmarks and captures nonlinear variability in shapes. In sections 5 and 6 we present some results and make a discussion on the relevance of our work.

\section{Modelling Intermittently Present Landmarks}

When modelling a class of objects with a PDM, it is needed the whole objects to be structured by the same number of landmark points. Nevertheless, in some examples, this is not possible due to the nature of the objects. Consider the class of objects in figure 1. There is a dependency between the aspect ratio of the external square and the size of the internal square. While the vertices of the external square moves along a straight line, the internal square appears, grows up, decreases and finally disappears. There is a quadratic dependency between the aspect ratio of the external square and the area of the internal one with a threshold that forces the internal square to disappear.

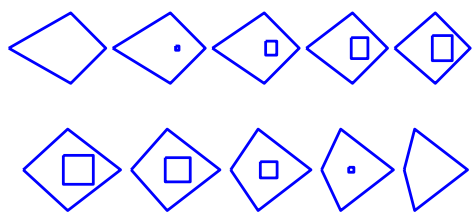

Fig. 1. Sombe objects of the training set. There are 16 points representing the figures with only the external square, and 32 the figures with both squares (there are 3 points along each line segment).

If we want to model this kind of variability with a PDM, it is needed a first process which is based on estimating the position of the missing landmarks in order to have non-missing points. We refer to this process as Data imputation 
(section 2.1). Then, a classical PDM might be applied. Thus for the training data after the imputation step $x=\left[x_{1}|\ldots| x_{N}\right]$ (where $x_{i}$ is the $i$ th training shape and $N$ the number of training shapes) we get a set of parameterizations $b=\left[b_{1}|\ldots| b_{d}\right]^{T}$ (where $b_{i}$ is the $i$ th principal mode and $d$ is the number of modes of the PDM). Nevertheless, the model would have lost the knowledge of the presence or absence of some of the landmarks. For this reason, it seems reasonable to define a model that considers this source of knowledge. We refer to this model as a Model for Intermittently-present Data. (section 2.2).

\subsection{Data Imputation}

In this section, two schemes for data imputation are depicted. In the first one, the missing data is replaced by the mean. Usually, this approximation underestimates the variance of the data. The second one was presented by Rogers and Graham [4 and it is known as Iterated PCA. The basic idea is to iteratively impute values in such a way as to retain the relationships present in the original data.

Mean Imputation In this model, the position $(\mathrm{x}, \mathrm{y})$ of the landmark points that are not present in some objects, are replaced by the mean (x,y) of the related landmark points that are present in the other objects.

We show in figure 2 the scattergram of the first two modes of variation of the PDM when the missing data is imputed with the mean. The vertical axe is represented by $b_{2}$ and codes the aspect of the external square, and the horizontal axe by $b_{1}$ that codes the size of the internal square. Low values (or negative) in $b_{2}$ represent big size and high values represent small size. It can be observed the nonlinear correlation between these two features. It represents the process of enlargment-reduction of the internal square throughout the process of varying aspect of the external square. The objects with originally no interior square are projected in the scattergram as two horizontal lines at the extrema of $b_{2}$. They are assigned to zero in $b_{1}$ since it corresponds to an interior square of mean size.

Figure 3 shows the reconstructed objects varying the two principal modes of variation between \pm 2 standard deviations (s.d.). The first mode, $b_{1}$, (first row) encodes the size of the interior square and the second mode, $b_{2}$, (second row) encodes the aspect of the external square. The linear PDM does lead to the generation of invalid shapes (not similar to the ones in the training set).

Iterated PCA . This is an iterative method to impute missing data developed by Rogers and Graham [4. There is an initial imputation based on the mean (section 2.1) and then the data is re-estimated iteratively until convergence. At the beginning, the first principal component of the data is used for re-estimation, and next subsequent principal components are added. The aim of this process is to imput the data in a way so as to retain the relationships represented by a new eigenvector at each step. The goal is to end up with an imputed data consistent with the data patterns represented by the eigenvectors. 


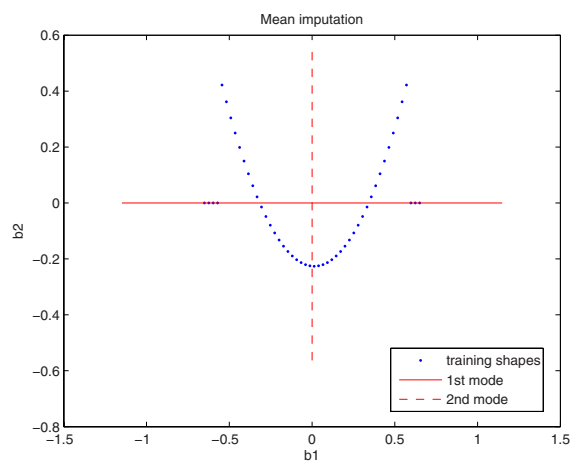

Fig. 2. Scattergram of the two main modes of variation. Data imputed with the mean.

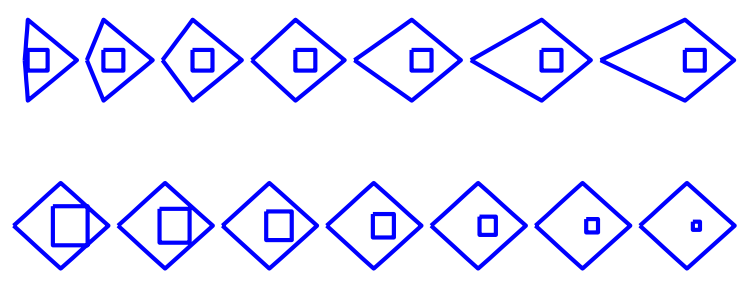

Fig. 3. Two principal modes of variation of PDM with mean imputation. From -2 to +2 s.d.

This method obtains practically the same results than the mean imputation using the example set given in figure 1. It is for this reason that we do not show explicitly the results. Since the imputation only affects to the size of the internal square, it can be seen as moving the points along $b_{2}$. Then, setting the points at zero in $b_{2}$ is according with the linear relationship defined by the first eigenvector $b_{1}$, and it is equivalent to put a mean-size internal square.

\subsection{A Model for Intermittently-Present Data}

In this work, we will use the model for intermittently-present data defined by Rogers and Graham called Structured Point Distribution Model (SPDM) 4]. The aim of this model is to build a PDM that deals with intermittently present landmarks.

Let $x$ be an initial training set composed by $N$ configurations of $k$ landmarks in two dimensions.

$$
x_{i}=\left[\left(x_{i 1}, y_{i 1}\right),\left(x_{i 2}, y_{i 2}\right), \ldots,\left(x_{i k}, y_{i k}\right)\right] .
$$


Let each configuration be represented by the data vector

$$
x_{i}=\left(x_{i 1}, x_{i 2}, x_{i 3}, \ldots, x_{i k}, y_{i 1}, y_{i 2}, y_{i 3}, \ldots, y_{i k}\right)^{T} .
$$

And let miss $(i)$ be the set of missing landmarks in the $i$ th configuration. The procedure to build an SPDM is the following:

1. Replace each missing value by a placeholder such as NaN (a computational representation of Not a Number).

$$
x_{i, \operatorname{miss}(i)}=y_{i, \operatorname{miss}(i)}=N a N, \quad 1 \leq i \leq N
$$

2. Align the configurations using the landmarks that are available with Generalized Procrustes Analysis [5].

3. Impute the missing landmarks by some imputation scheme (see Section 2.1 for further details).

Then, an initial training shape $i$ with $\operatorname{miss}(i)=\{2,3\}$ becomes

$$
\hat{x}_{i}^{\prime}=\left(x_{i 1}^{\prime}, \hat{x}_{i 2}, \hat{x}_{i 3}, \ldots, x_{i k}^{\prime}, y_{i 1}^{\prime}, \hat{y}_{i 2}, \hat{y}_{i 3}, \ldots, y_{i k}^{\prime},\right)^{T},
$$

where primed elements are aligned and hat elements are imputed.

4. Perform a shifting and scaling in order to make the data $\hat{x}^{\prime}$ to lie between 0 and 1.

$$
\tilde{x}_{i}=\frac{x_{i}-x^{\min }}{x^{\max }-x^{\min }}, \quad 1 \leq i \leq N,
$$

where $x^{\max }$ and $x^{\text {min }}$ are the vectors with the maximum and minimum values of $\hat{x}^{\prime}$ for each dimension, respectively.

This shifting and scaling is to avoid problems associated with shape and structure being measured on different scales. It responds to the consideration of treating shape and structure as equally important.

5. Build a classical PDM [2], getting a parameterization $b^{d}$ for the normalized training shapes

$$
\tilde{x}=\bar{x}+\Phi b^{d}
$$

where $\Phi$ are the eigenvectors, and $\bar{x}$ is the procrustes mean shape [5].

While this gives us a model of shape variation, we have lost the structural information about which landmarks are present and which are not.

6. Augment the shape vector with an structure vector informing about the presence or absence of each landmark.

$$
x_{i}^{s}=\left(x_{i 1}^{s}, x_{i 2}^{s}, \ldots, x_{i k}^{s}\right)^{T},
$$

where $x_{i j}^{s}=\{0,1\}$ depending on whether the $j$ th landmark of the $i$ th shape $\left(x_{i j}, y_{i j}\right)$ is present or not. 
7. Apply PCA on these structure vectors in order to reduce the redundancy, obtaining a reduced parameter vector $b^{s}$ representing the structure vector

$$
x^{s}=\overline{x^{s}}+P b^{s},
$$

where $P$ are the eigenvectors of the covariance matrix of the structural data $x^{s}$, and $\overline{x^{s}}$ is the mean structure vector of the $N$ samples.

8. Build a combined model of shape and structure. i.e., for each training shape generate a concatenated vector

$$
b=\left(\begin{array}{c}
b^{d} \\
b^{s}
\end{array}\right)=\left(\begin{array}{c}
\Phi^{T}(\tilde{x}-\bar{x}) \\
P^{T}\left(x^{s}-\bar{x}^{s}\right)
\end{array}\right)
$$

9. Apply PCA again to obtain a combined model of shape and structure

$$
b=Q c
$$

where $Q$ are the eigenvectors and $c$ is a vector of structural shape parameters controlling both the position and presence or absence of the shape points. Since the shape and structural parameters have zero mean, $c$ does too.

Note that the linear nature of the model allows to express the shape and its structural information directly as functions of $c$

$$
\tilde{x}=\bar{x}+\Phi Q_{d} c \quad, \quad x^{s}=\bar{x}^{s}+P Q_{s} c
$$

where

$$
Q=\left(\begin{array}{l}
Q_{s} \\
Q_{d}
\end{array}\right)
$$

and the original shape is given by

$$
x=\tilde{x}\left(x^{\max }-x^{\min }\right)+x^{\min }
$$

An example shape can be synthesised for a given $c$ by generating the shape from the vector $x$ and removing those landmarks according to $x^{s}$ and a given threshold.

The SPDM like the PDM is a generative model, i.e., given a parameter vector we can recreate the structure vector for a particular instance. The main drawback comes from the fact of representing a binary process (presence or absence) by a linear model. Thus, to recover binary parameters in the reconstructed structure vector, a threshold representing the probability of presence or absence, must be applied.

By the inclusion of the structure vector $x^{s}$ we allow for arbitrary patterns of inclusion/exclusion.

Figure 4 shows the scattergrams obtained using this model and imputing the data with the mean. The horizontal axes of both scattergrams, $b_{1}$, represent the mode that captures the variability in the presence or absence of the points. Here the objects represented by the points at $b_{1} \simeq-0.5$ have the interior square 


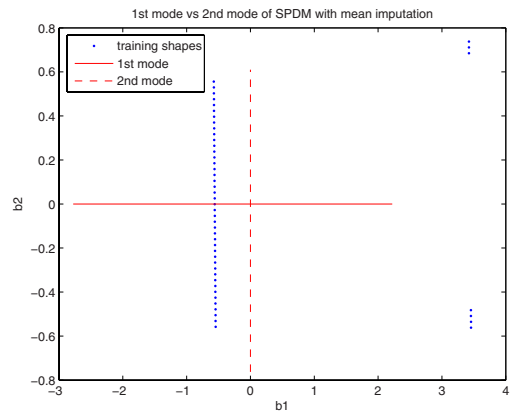

(a)

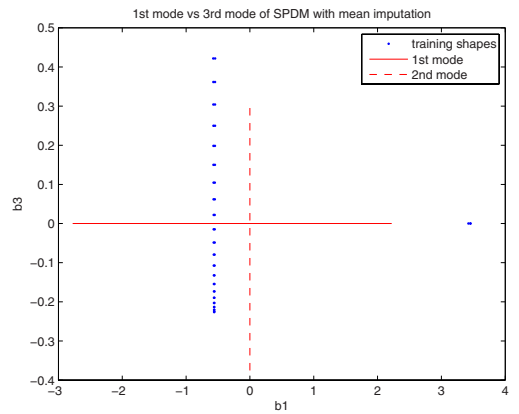

(b)

Fig. 4. Scattergrams of the three main modes of variation. Data imputed by the mean and using the SPDM.

present, and the those at $b_{1} \simeq 3.5$ have not. The mode that captures the variability in the aspect ratio of the exterior square, $b_{2}$, is represented by the vertical axe of figure (a) and the mode that captures the variability in the size of the interior square, $b_{3}$, is represented by the vertical axe of figure (b).

Figure 5 shows the objects generated by varying the mode $b_{1}$ of the SPDM between \pm 2 s.d., which represents the presence or absence of the internal square. The objects generated by varying the mode that represents the size of the internal square and by varying the mode that represents the aspect of the external square are not shown due to they are identical to the ones generated by a PDM with a mean imputation (figure 3).

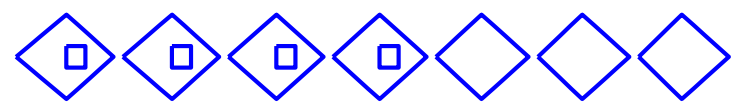

Fig. 5. First principal mode of variation of SPDM. Data with mean imputation.From -2 to +2 s.d.

\section{Modelling Nonlinear Dependencies}

When the modes of variation obtained from the objects of a class have nonlinear dependencies, as it is the case of our example, the model specificity obtained by a classical PDM is very poor (figure 31). To solve this problem, Sozou et al. defined a nonlinear generalization of PDM called Polynomial Regression Point Distribution Model (PRPDM) 3. The aim is to model all the modes $b_{i}$ as nonlinear functions of a given mode: $b_{i}^{*}=f_{i}^{k}\left(b_{k}\right)$, where $b_{k}$ is the mode being fitted, $f_{i}^{k}(\bullet)$ is the polynomial that models the $i$ th mode as function of the $k$ th mode, and $b_{i}^{*}$ are the modelled values. Next, the new residuals $b_{i}^{\text {new }}$ are computed as the difference between the data values of $b_{i}$, and the modelled values of $b_{i}^{*}$ : 


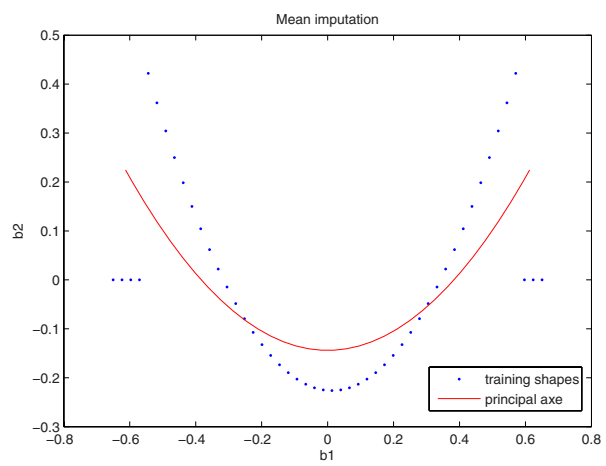

Fig. 6. Scattergram of the linear decomposition of the training data with mean imputation. The first principal mode of variation of PRPDM is superimposed.

$b_{i}^{\text {new }}=b_{i}-b_{i}^{*}$. This procedure is repeated until there are no more residuals or until we have explained the desired variance. Note that at each step one mode is removed since we ignore the mode being fitted $b_{k}$. At each step we append the values of $b_{k}$ into a new $b^{\prime}$, and store the set of functions $f_{i}^{k}(\bullet)$.

This model assumes that there is no missing data. In our data set, there are some missing points. In this case, the missing points in these experiments have been imputed using the mean imputation scheme presented in section 2.1.

Figure 6] shows the scattergram of the first two modes of the linear decomposition of the training data with mean imputation. The data is the same than the one presented in figure 2, but the first automatically-obtained polynomial mode (that fits $b_{1}$ to $b_{2}$ ) is superimposed. The polynomial mode do not adjust to the data due to the noise introduced by the mean imputation and so, the model does not retain properly the variability of the training set.

Figure 7 shows the objects generated varying the first mode of the PRPDM between \pm 1.5 s.d.

The generated objects are more similar to the original ones (figure 1) than the ones generated by the linear PDM (figure 3) but the results are not good enough. In the following section, we present a new model with the aim of solving the two main problems described in the above sections. It has to capture the nonlinear variability in a more accurate way and also, it has to capture the presence or absence of some data.

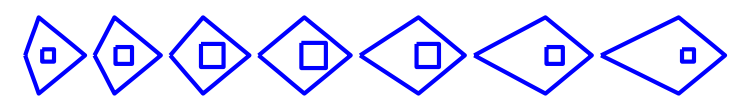

Fig. 7. First principal mode of PRPDM. Data with mean imputation. From -1.5 to +1.5 s.d. 


\section{Nonlinear Modelling of Intermittently Present Data}

The main idea of this new model is to imput the missing data according to the nonlinear relationships of the present data. Thus, we expect to obtain a better approximation of the polynomial regression in the present data. To that aim, we have defined a new imputation model called Iterated Nonlinear PCA inspired in the model Iterated PCA explained in section 2.1. Moreover, and with the aim of not loosing the knowledge of the presence or absence of the data, we have defined a new model for intermittently present data inspired in the one commented in section 2.2. The new model is useful to model nonlinear variability as the PRPDM (section [3) and intermittently present data as in the SPDM (section 2.2). For this reason, we have called Polynomial-Regression Structured Point Distribution Model (PR-SPDM).

\subsection{A New Imputation Scheme: Iterated Nonlinear PCA}

We have developed this method as an adaptation of iterated PCA, intended to fit to our purposes. The idea is to impute the missing data according to the nonlinear relationships present in the original data.

The algorithm can be described with the following equations:

$$
\begin{gathered}
\left(P_{x d}, \mu_{x}, b_{x d}\right)=p c a(x, d) \\
\left(A_{x m}, c_{x m}\right)=\operatorname{prpca}\left(b_{x d}, m\right) \\
\hat{b}_{x d}^{m}=\operatorname{prreconstr}\left(c_{x m}, A_{x m}\right) \\
\hat{x}=\mu_{x}+P_{x d} \cdot \hat{b}_{x d}^{m} \\
x_{i, m i s s(i)}=\hat{x}_{i, m i s s(i)}
\end{gathered}
$$

where $x$ is the original data, $x_{i j}$ is the $j$ th value in example $i, p c a$ is a function that computes the first $d$ principle components $P_{x d}$ and the mean $\mu_{x}$, together with the associated reconstruction parameters $b_{x d}$ for each training example. prpca is a function that computes the associated projections $c_{x m}$ of the parameters $b_{x d}$ to the space spanned by the first $m$ principal polynomial modes $A_{x m}$. prreconstr is the function that reconstructs the $d$ dimensional data $\hat{b}_{x d}^{m}$ from the projections $c_{x m}$ using the first $m$ polynomial axes $A_{x m}$. miss $(i)$ is the set of variables missing in example $i$ and $\hat{x}_{i, m i s s(i)}$ is the set of estimated missing values from $x_{i}$.

It consists on a two-step procedure. First $b_{x d}$ is obtained by the projection of $x$ onto the first $d$ principal linear components (eq. (9)). Next these data are used to compute the nonlinear modes of variation, and are then projected using the first $m$ of these modes, obtaining $c_{x m}$ (eq. (10)). Then, we reconstruct the 
original data $\hat{x}$ (eqs. (11)-(12) ), and replace the imputed values (eq. (13)). The algorithm starts by mean imputation of the missing data, $m$ is set to 1 , and cycle through equations (9)-(13) until convergence. Next, we increment $m$ by 1 and repeat the procedure. At each step the data is according to the nonlinear relationships retained by the first $m$ principal nonlinear components. The process is repeated until $\frac{\Sigma \sigma_{x m}^{2}}{\sigma_{x}^{2}}>0.9$, where $\sigma_{x m}^{2}$ is the variance of the $m$ th mode and $\sigma_{x}^{2}$ is the variance of all modes.

Figure 8 shows the scattergram of the linear decomposition of the training data with iterated nonlinear PCA imputation, together with the first principal mode of the PRPDM. We have set $d=3$ in the execution of the algorithm since the $99 \%$ of the variance is explained by 3 eigenvectors. Making use of this imputation scheme, we observe two main properties. First, the objects with missing data (points such that $b_{1}<-0.6$ and $b_{1}>0.6$ ) are according to the first principal nonlinear mode, instead of having a mean-size square (which is according to the first principal linear mode). And second, the obtained polynomial approximates better the whole dataset, which means that the model is more specific.

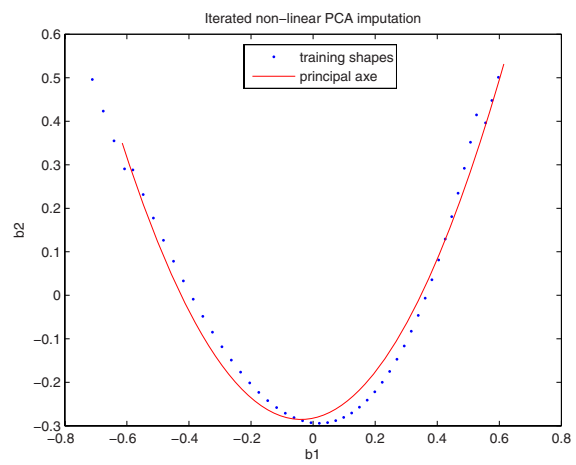

Fig. 8. Scattergram of the linear decomposition of the training data. Data imputed by the iterated nonlinear PCA and modelled by the PRPDM. The first principal mode of the PRPDM is superimposed.

Figure 9 shows the objects generated varying the first mode of the PRPDM between \pm 1.5 s.d.

In this example the nonlinear models are more compact, since only one mode of variation is needed to generate good approximations of the objects. This is due to the fact that the nonlinear dependency between the size of the interior square and the position of the exterior one is properly acquired by PRPDM. Moreover, when imputing with iterated nonlinear PCA, the generated objects are closer to the original ones than the ones generated by any of the other models presented before. Nevertheless, in the generated objects, the interior square is 


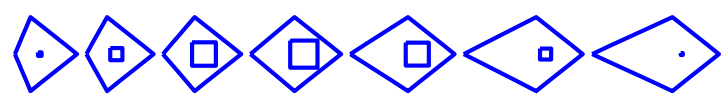

Fig. 9. First principal mode of PRPDM. Data imputed with iterated nonlinear PCA. From -1.5 to +1.5 s.d.

always present. The knowledge of the presence or absence of some points has not been considered. This is the topic of the following section.

\subsection{A New Model for Intermittently-Present Data: PR-SPDM}

This model is useful to capture sets of objects with missing data (or landmarks) and also, captures the nonlinearity between the relationships of the landmarks.

Let $x$ be an initial training set composed by $N$ configurations of $k$ landmarks in two dimensions. And let miss $(i)$ be the set of missing landmarks in the $i$ th configuration.

The procedure to build a PR-SPDM is the following:

1. Follow the steps 104 of the procedure to build an SPDM in Section 2.2 After these steps, we end up with an aligned set of shapes with the missing landmarks imputed, and this whole set normalized to lie between 0 and 1 . We denote this set as $\tilde{x}$.

2. Build a classical PRPDM with the dataset $\tilde{x}$, as indicated in Section 3 and get the nonlinear parameterizations $b_{p r}^{d}$ for each shape $\tilde{x}_{i}$.

3. Augment the shape vector with an structure vector $x_{i}^{s}$ informing about the presence or absence of each landmark as in step 6 of the SPDM procedure in section 2.2

4. Apply PCA to the structure vectors $x_{i}^{s}$ in order to reduce the redundancy, and get an eigenvectors matrix $P$ for the structure parameterization as in step 7 of the SPDM procedure in section 2.2 .

5. Build a concatenated vector of shape and structural parameterizations

$$
b=\left(\begin{array}{c}
b_{p r}^{d} \\
b^{s}
\end{array}\right)
$$

6. Apply PCA again to obtain a combined model of shape and structure

$$
b=Q c
$$

where $Q$ are the eigenvectors and $c$ is a vector of structural shape parameters controlling both the position and presence or absence of the shape points. Since the shape and structural parameters have zero mean, $c$ does too.

Note that a shape and its structural information can be recreated for a given $c$.

$$
b_{p r}^{d}=Q_{d} c \quad, \quad x^{s}=\bar{x}^{s}+P Q_{s} c
$$


where

$$
Q=\left(\begin{array}{l}
Q_{s} \\
Q_{d}
\end{array}\right)
$$

and $\tilde{x}$ is computed reconstructing the PRPDM parameters.

Finally, the original shape is given by

$$
x=\tilde{x}\left(x^{\max }-x^{\min }\right)+x^{\min }
$$

An example shape can be synthesised for a given $c$ by generating the shape from the vector $x$ and removing those landmarks according to $x^{s}$ and a given threshold.

Figure 10 shows the scattergrams of the PR-SPDM with mean (a) and iterated nonlinear PCA (b) imputation of the missing data. The horizontal axes, $b_{1}$, encode the inclusion and exclusion of points. The vertical axes, $b_{2}$, correspond to the first nonlinear modes of the PRPDMs.

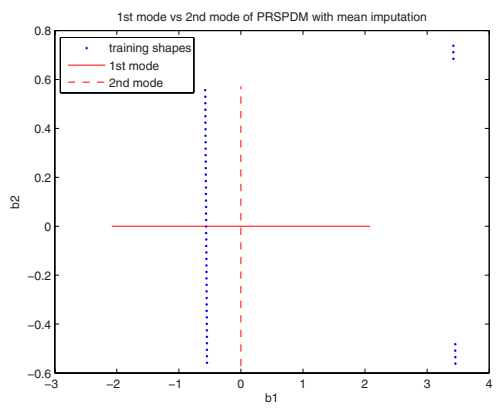

(a)

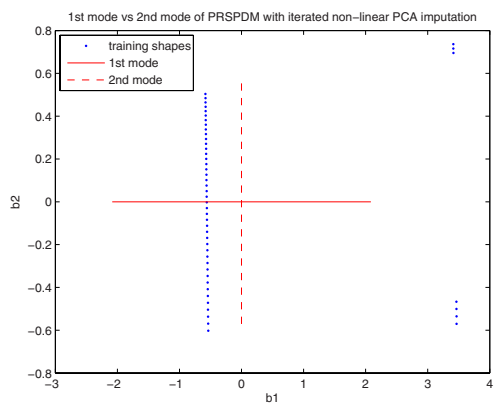

(b)

Fig. 10. Scattergrams of the two first principal components of PR-SPDM for data with (a) mean imputation, and (b) iterated nonlinear PCA imputation

Figure 11 shows the objects generated by varying the first mode of the PRSPDM between \pm 1.5 s.d. for data with both mean imputation and iterated nonlinear PCA imputation. As in the case of SPDM, the first principal mode of PR-SPDM represents the inclusion or exclusion of points. The remaining two modes capture deformations in shape as seen in figures 7 for mean imputation and 9 for nonlinear PCA imputation.

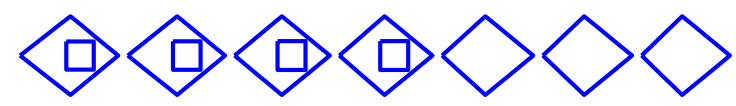

Fig. 11. First principal mode of PR-SPDM. It is the same for mean imputation and iterated nonlinear PCA imputation. From -1.5 to +1.5 s.d. 


\section{$5 \quad$ Evaluation Experiments}

We have perturbed the $(x, y)$ coordinates of the training images with noise. We calculate the mean square error between the reconstructed objects using each of the models described, and the original images. We present the results in two plots corresponding to objects composed by one and two squares (figs 12a and 12,b). We use the first two principal modes of each model.

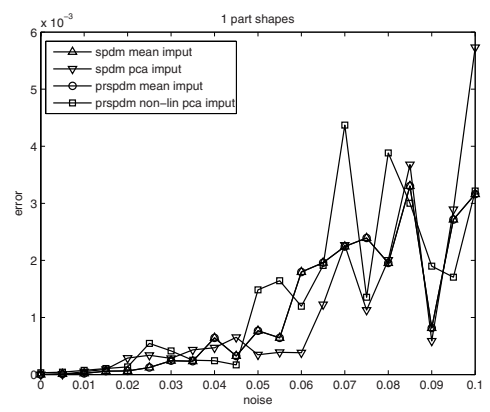

(a)

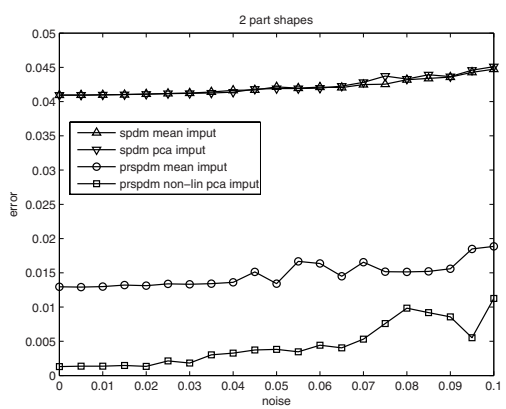

(b)

Fig. 12. Reconstruction errors for, (a) 1-part and, (b) 2-parts objects

While the differences of the objects which have only one square are negligible, models using PR-SPDM do present better results in comparison with ones using SPDM for objects which have the two squares. This is because two modes are enough for the PR-SPDM to explain the three sources of variability (presence/absence, aspect ratio of external square and size of internal square), while SPDM only explains the first two with the same number of modes. Between the nonlinear models, the one with iterated nonlinear PCA imputation achieves clearly the best results.

\section{Conclusions and Future Work}

We have presented a new scheme for imputing missing data and also to model the presence or absence of the data. The imputation scheme, called Iterated nonlinear PCA, aims to generate the missing data according to the nonlinear relationships of the present data set. The model defined to capture the presence or absence of the data is called Polynomial-Regression Structured-Point Distribution Model (PR-SPDM). Applying this model, a new variability mode appears to represent the presence or absence of data. Results show that the imputed data plays an important role in the resulting model making it more specific. Moreover, our model achieves the best representation of the data set and we believe that it is portable to real examples, i.e. medical images. We leave this study as a future 
work. It is a topic of future research to model the clearly nonlinear relationships between inclusion/exclusion of landmraks and shape deformation into nonlinear modes of variation of a PR-SPDM.

\section{References}

1. Kass, M., Witkin, A., Terzopoulos, D.: Snakes - active contour models. International Journal of Computer Vision 1(4), 321-331 (1987)

2. Cootes, T.F., Taylor, C.J., Cooper, D.H., Graham, J.: Active shape models - their training and application. Computer Vision and Image Understanding 61(1), 38-59 (1995)

3. Sozou, P.D., Cootes, T.F., Taylor, C.J., Dimauro, E.C.: Nonlinear generalization of point distribution models using polynomial regression. Image and Vision Computing 13(5), 451-457 (1995)

4. Rogers, M., Graham, J.: Structured point distribution models: Modelling intermittently present features. In: Proceedings of the British Machine Vision Conference, vol. 1, pp. 33-42 (2001)

5. Goodall, C.: Procrustes methods in the statistical-analysis of shape. Journal of the Royal Statistical Society Series B-Methodological 53(2), 285-339 (1991) 